\title{
Prevalence of Otorhinolaryngeal Malignancies in Thanjavur Delta Region-A Prospective Study
}

\author{
Ganesh Kumar Balasubramaniam, Ramesh Babu Kalyanasundaram, \\ Ramanathan Thirunavukkarasu, Gitanjali Narendran, Jagadeesh Natarajan \\ Department of ENT and Head and Neck Surgery, Thanjavur Medical College, Thanjavur, India \\ Email: drganeshkumarb@gmail.com
}

Received 30 December 2015; accepted 5 March 2016; published 8 March 2016

Copyright @ 2016 by authors and Scientific Research Publishing Inc.

This work is licensed under the Creative Commons Attribution International License (CC BY).

http://creativecommons.org/licenses/by/4.0/

(c) $\underset{\mathrm{EY}}{0}$ Open Access

\begin{abstract}
Malignancy of otorhinolaryngeal region is the $6^{\text {th }}$ common malignancy worldwide and nowadays the incidence is increasing in developing countries like India. This malignancy contributes to $23 \%$ of male and 6\% of female population of India [1]. Malignancies included in this study are those of oralcavity, oropharynx, nasopharynx, hypopharynx, larynx, thyroid, nose, paranasal sinuses and salivary gland tumors. This study was conducted in the Department of Otorhinolaryngology and Head and Neck Surgery, Thanjavur Medical College, Thanjavur, Tamilnadu India, over a period of 2 years from August 2013 to February 2015. Histopathologically confirmed cases alone were taken for this study. In our study the laryngeal and hypopharyngeal malignancies outnumber the other regions. Males are more commonly affected and the age group of around 40 - 50 years is more commonly involved. Smoking and alcohol intake plays a major etiological factor. Cancer awareness programmes and health education regarding risk factors can help to reduce the incidence of malignancies in our Thanjavur delta region.
\end{abstract}

\section{Keywords}

Head and Neck Cancers, Larynx, Hypopharynx, HPV, Squamous Cell Carcinoma, Smoking and Alcohol, Tracheostomy, Radiotherapy

\section{Introduction}

Malignancy of the head and neck and face is more common in Asia, especially in India. The laryngeal and hypopharyngeal malignancies are more common in heavily industrialized areas. Most of the patients are more than 
40 years males, due to tobacco and alcohol use. The effects are dose, duration and variety dependent. Cigar and Beedi are more carcinogenic compared to filter cigarette. Among the alcoholic beverages, Arrack and Rum are more carcinogenic than beer. When smoking is combined with alcohol there will be $15 \%$ increased incidence of malignancy. Cessation of smoking leads to gradual reduction in risk by $70 \%$ after 10 years [2]. Burning of tar produces methylcholanthrine and benzanthracene which are broken down by aryl-hydrocarbon hydroxylase into carcinogenic epoxides which bind to DNA.

The p53 tumour suppressor gene mutation plays a major role in head and neck tumours. This mutation is present in the tumours of 58\% of patients with history of both smoking and alcohol, and 33\% of patients with history smoking alone. Grapes and wine contain Resveratrol, a cancer chemopreventive agent. Glutathione S transferase plays a major role in head and neck tumours [3]. Nickel and chromate dust are major risk factors for nasal and paranasal sinus malignancy, hardwood dust for adenocarcinoma of paranasal sinuses and nitrosamines for nasopharyngeal malignancies.

Paterson-Brown-Kelly syndrome (iron deficiency anaemia, glossitis, koilonychia, achlorhydria and upper oesophageal web) is present in $4 \%-16 \%$ of postcricoid malignancies. If we identify this syndrome at an early stage, it can be reversed by iron and vitamin B complex therapy. Human papilloma viruses (HPV) type 6, 18 and 33 are carcinogenic for laryngeal tumours and Ebstein Barr Virus (EBV) for nasopharyngeal carcinomas [2]. In our study we enquired more about the personal habits, family history and the working environment because all these play a major role.

\section{Aims of the Study}

1) To assess the prevalence of otorhinolaryngeal malignancy in Thanjavur Medical College Area.

2) To identify the type of malignancy which is most prevalent.

By the end of the study we will plan to conduct cancer awareness program for the rest of the population to minimize the incidence of this type of malignancies.

\section{Materials and Methods}

A prospective study on prevalence of malignancies in the otorhinolaryngeal sites like oral cavity, oropharynx, nasopharynx, larynx, hypopharynx, nose and paranasal sinuses was conducted in our Department of Otorhinolaryngology and Head and Neck Surgery in Thanjavur Medical College, Thanjavur during the period of August 2013 to February 2015 (18 months). Around 220 newly diagnosed cases of head and neck malignancy were taken for the study. Thyroid and Salivary gland tumours were not included in this study. From the time of admission to the final stage of management of the head and neck tumours such as surgery or radiotherapy or chemotherapy, all the patients were followed regularly. Only histopathologically proved cases were included.

\section{Results}

In our study we came across 220 cases of Otorhinolaryngology malignancies. This table shows the distribution of all types of head and neck malignancies (Table 1). This incidence is similar to the Abhinandhan bhattacharjee study [4].

Table 1. Topographic distribution of head and neck malignancies.

\begin{tabular}{ccc}
\hline Type of tumour & No of cases & Percentage \\
\hline Larynx & 67 & $30.45 \%$ \\
Hypopharynx & 56 & $25.45 \%$ \\
Oropharynx & 39 & $18 \%$ \\
Oral cavity & 17 & $8 \%$ \\
Nose \& PNS & 18 & $8.18 \%$ \\
Nasopharynx & 14 & $6.36 \%$ \\
Others & 9 & $4 \%$ \\
\hline
\end{tabular}


Among the laryngeal malignancies the supraglottic subsite (41 cases) was involved more than the glottis (25 cases) and subglottis (1 case) (Figure 1). All the laryngeal malignancies are common in the male sex only. This is mostly due to their habits of smoking and alcohol and also multiple sexual partners mainly for the HPV spread tumours. The most common age groups involved are around 40 - 60 years.

Among the hypopharyngeal growths, postcricoid region (23 cases) is involved more commonly than Pyriform sinus (20 cases) and Posterior Pharyngeal wall (13 cases) malignancies (Figure 2). Post cricoid malignancy is more common in females. Pyriform sinus malignancy is common in both sexes. Age groups commonly involved are 40 - 60 years.

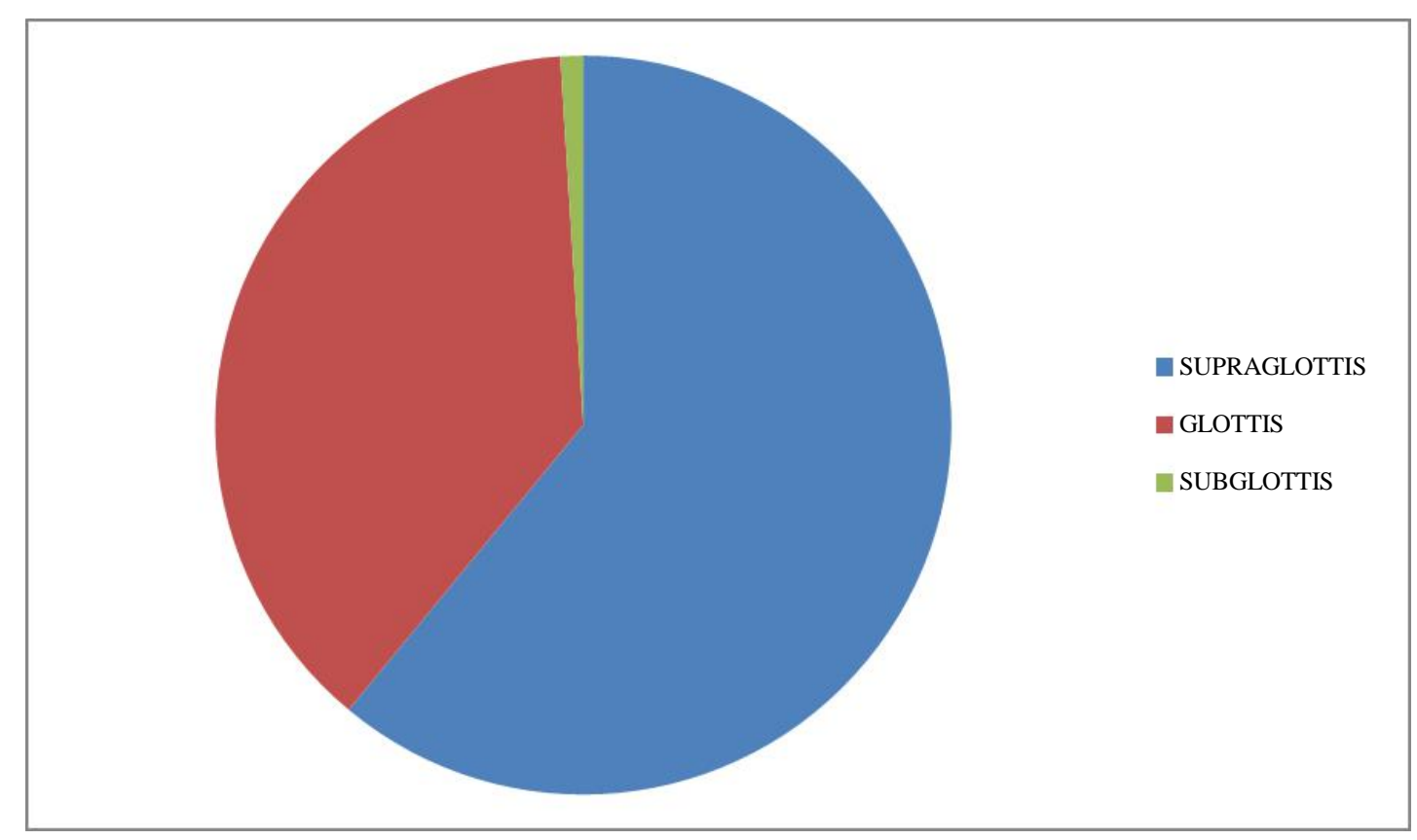

Figure 1. Distribution of laryngeal malignancies.

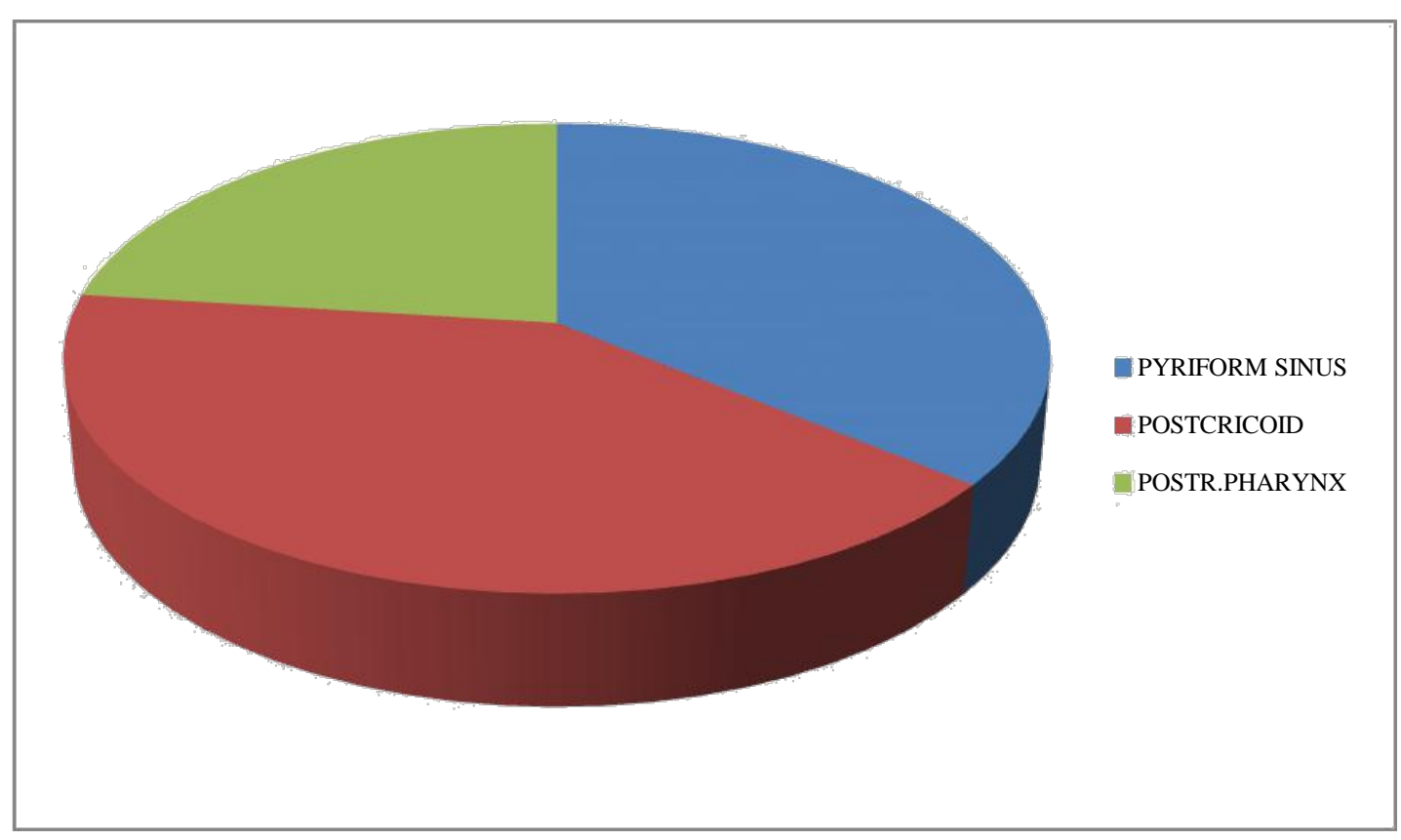

Figure 2. Distribution of hypopharyngeal malignancies. 
Among the oropharyngeal malignancies, posterior one third of tongue, valleculae and tonsil are common subsites. They are predominantly common in males due their habit of pan and betelnut chewing and oral sex with multiple partners which is responsible for spread of HPV, the main etiology for oral and oropharyngeal malignancies [5]. They are more commonly seen in 40 - 60 years age group. But in our study and also the MMTR study, the base of the tongue and valleculae are common subsites. Oral cavity malignancy was found in 17 cases. Anterior two third of tongue and hard and soft palate are common subsites. Male sex involvement is due to the habit of chewing pan gutka, reverse smoking and oral sex. Malignancy of the nasal cavity was found in 10 cases. No sex predilection was seen in these tumours. Nasopharyngeal malignancy was identified in 14 cases. Males were more commonly affected than females. Paranasal Sinus malignancy was seen in 8 cases. It is more common in males and above the age of 60 years. Maxillary sinus is more commonly involved than other sinuses.

The rest of the tumours were lymphoma (1), Parapharyngeal mass (1), Plummer Wilson Syndrome (2), Papilloma of the buccal mucosa (2) and 2 cases of Oesophageal malignancy and one case of mandible malignancy.

In our study around $70 \%$ of cases had both alcohol and smoking as risk factors for all types of malignancies, which is similar to William J Blot and Joseph KMC Laughlin (1998) article which was published in American association of cancer research article [6].

\section{Discussion}

In our institutional study the laryngeal malignancies are more in number followed by hypopharyngeal and oropharyngeal malignancies. Various Indian statistical studies like those of Chakraborty, Ray Chowdury and MMTR the supraglottic subsite is common [7]. So our study coincides with these studies. But in Lederman's study, Powel \& Robin and Thawley studies, glottis is the most common subsite for the laryngeal tumours.

In our study, the most common presentation of supraglottic tumours was odynophagia and a feeling of lump in the throat. Many patients presented with neck secondaries and some patients presented with stridor. On these occasions we proceeded with tracheostomy followed by direct laryngoscopy and biopsy. Glottic tumours presented with hoarseness of voice and in some cases with respiratory distress. They were also treated with tracheostomy followed by biopsy. Subglottic tumour presented in the early stage with stridor and was managed by tracheostomy and biopsy.

Among Hypopharyngeal malignancies, postcricoid subsite is common followed by pyriform sinus and posterior pharyngeal wall in our study, which is parallel to Jones \& Stell Liverpool study series. In our study cancer of oesophagus had a lesser incidence compared to Liverpool study. All Hypopharyngeal tumours presented with difficulty in swallowing. Postcricoid growth with stridor was managed by emergency airway management followed by biopsy.

Oral cavity malignancy is the $11^{\text {th }}$ common tumour globally. In India, gingivo-buccal complex (alveolar ridge, gingivobuccal sulcus and buccal mucosa) is the commonest subsite. But in our study, base of tongue and valleculae are common subsites. Recent survey shows that oral cavity malignancy forms the leading cause of mortality (around 22.9\%) in cancer related death [8].

Nasopharyngeal malignancy presented with neck swelling and ipsilateral aural fullness. Nasal and paranasal sinus malignancies presented with nasal obstruction, bleeding per nose and facial asymmetry. In these tumours, we proceeded with biopsy.

Most of the tumours presented in the late stage except the glottic tumours. More than $60 \%$ of cases were in stage II \& III. Histopathological report revealed Squamous Cell Carcinoma in $85 \%$ of tumours (which is similar to MMTR-Madras Metropolitan Tumour Registry) and its variants like Verrucous carcinoma, Basaloid carcinoma and some cases were Adenocarcinoma [9].

All squamous cell carcinomas were treated with surgery, radiotherapy and chemotherapy depending on the stage and the patient's general condition. Many cases required prophylactic neck dissection even without the nodal involvement. All stage IV tumors were treated on palliative and symptomatic basis.

\section{Conclusions}

This study shows the distribution pattern of various types of head and neck malignancy in Thanjavur Medical College region. The results are almost similar to many National and International studies. Smoking and Alcohol are important risk factors. These habits are slowly increasing in the female population also nowadays. So awareness programmes, counseling and IEC activities are essential for the patients especially in a developing 
country like India. Health education about the side effects of betel nut chewing, pan gutka usage and reverse smoking and oral sex is mandatory. Preventive activities regarding the side effects of these things should be carried out regularly [10].

Most of the head and neck tumours are identified in the late stages only, so curative treatment is not possible. So cancer awareness programmes, screening for malignancies by camps will be useful to identify the tumours at an early stage and to get a better 5-year survival rate [11].

\section{References}

[1] Mehrotra, R., Singh, M. and Singh, M. (2005) Trends of Prevalence and Pathological Spectrum of Head and Neck Cancers in North India. Indian Journal of Cancer, 42, 89-93.

[2] Yeole, B.B. (2007) Trends in Incidence of Head and Neck Cancers in India. Asian Pacific Journal of Cancer Prevention, 8, 607-612.

[3] Blot, W.J. and Laughlin, J.K.M.C. (1998) Study of Association of Smoking and Alcohol in Upper Aerodigestive Tract Malignancies. American Association on Cancer Research, Philadelphia.

[4] Sangaranarayanan Gopalakrishnan, Praveenkumar. (2003) Epidemiological Study on Head and Neck Tumours. Otolaryngology Online Journal.

[5] Choudhry, R. (2002) Topographical Distribution of Laryngeal Carcinoma-A Study of 50 Cases. Indian Journal of Otolaryngology and Head and Neck Surgery, 54, 291-293.

[6] Battacharjee, A., Chakraborthy, A. and Purkaystha, P. (2006) Prevalence of Head and Neck Cancers in the North East India. Indian Journal of Otolaryngology and Head and Neck Surgery, 58, 15-19.

[7] Westra, W.H. (2009) The Changing Face of Head and Neck Cancer in $21^{\text {st }}$ Century: The Impact of HPV in Pathology of Oral Cancer. Head and Neck Pathology, 3, 78-81. http://dx.doi.org/10.1007/s12105-009-0100-y

[8] More, Y. and D’Cruz, A.K. (2013) Oral Cancer: Review of current Management Strategies. The National Medical Journal of India, 26, 152-158.

[9] Roland, N.J. (2008) Staging of Head and Neck Cancer. Scott Brown’s Otorhinolaryngology, Head and Neck Surgery. 7th Edition, Hodder Arnold, London, Volume 2, 2359-2371.

[10] Gangly, I. and Patel, S.G. (2012) Epidemiology and Prevention of Head and Neck Cancers. In: Watkinson, J. and Gilbert, R.W., Eds., Stell and Maran's Textbook of Head and Neck Surgery and Oncology, 5th Edition, CRC Press, Boca Raton, 9-14.

[11] Shantha, V. and Swaminathan, R. (2010) MMTR-Madras Metropolitan Tumour Registry. National Cancer Registry Program, ICMR, Cancer Institute (W.I.A), Chennai. 